\title{
Relación entre estilos parentales, intensidad psicopatológica y tipo de sintomatología en una muestra clínica adolescente
}

\author{
José M. Antón-San Martín ${ }^{1 *}$, David Seguí-Durán ${ }^{1}$ Laura Antón-Torre ${ }^{2}$ y Ana Barrera-Palacios ${ }^{1}$ \\ ${ }^{1}$ Unidad de Salud Mental Comunitaria. UGCSM Área Sanitaria Norte de Málaga. Hospital de Antequera \\ ${ }^{2}$ Asociación Antequerana de Mujeres Mastectomiz̨adas (AAMM). Antequera (Málaga, España)
}

\begin{abstract}
Resumen: Se realizó una investigación con el objetivo de analizar la relación entre los estilos parentales percibidos, la intensidad de la psicopatología presentada y las dimensiones sintomáticas internalizante-externalizante en una muestra clínica de adolescentes con edades comprendidas entre los 13 y los 18 años. Los resultados hallados indican que la intensidad psicopatológica está asociada con la edad, a mayor edad mayor intensidad, y con el género femenino. Solamente dos dimensiones del estilo parental percibido se hallaron relacionadas con la intensidad psicopatológica: el control psicológico de forma negativa y el humor, de forma positiva. $\mathrm{Al}$ contrario que en otros estudios consultados, la dimensión control psicológico apareció vinculada con sintomatología externalizante y no con la internalizante.

Palabras clave: Adolescencia; estilos parentales; psicopatología; internalizante-externalizante.
\end{abstract}

Title: Relationship between parenting styles, psychopathological intensity and type of symptoms in a adolescents clinical sample.

Abstract: An investigation was conducted to analyze the relationship between perceived parenting styles, presented intensity psychopathology and externalizing-internalizing dimensions in a clinical sample of adolescents aged 13 to 18 . The results found indicate that the psychopathological intensity is associated with age, with older age and over intensity; and with female gender. Only two dimensions of perceived parenting style were found related to psychopathological intensity: psychological control negatively and humor positively. As opposed to other studies consulted, the psychological control dimension appeared linked to externalizing symptoms and not with internalizing.

Key words: Adolescence; parenting styles; psychopathology; internalizingexternalizing symptoms.

\section{Introducción}

La familia es un sistema dinámico sometido a procesos de desarrollo y a múltiples transiciones o periodos de cambio inherentes a su evolución (Carter y McGoldrick, 1980; Falicov, 1991). Atraviesa en su desarrollo diversos momentos de diferente complejidad en cuanto a los procesos de transformación implicados, de acuerdo, entre otros factores, a los cambios que tienen lugar en algunos de sus componentes. La adolescencia es un ejemplo de estos procesos de mutación provocados, entre otros, por los cambios biológicos, psicológicos y sociales; por la gran influencia de sistemas extrafamiliares en el funcionamiento familiar, así como las modificaciones en la interacción entre padres e hijos en cuanto a la renegociación en los temas de control, de los límites y de la individuación-diferenciación. Se demanda más autonomía e independencia. De la marcada jerarquización propia de la niñez se pasa a la mayor igualdad y equilibrio de poder que caracterizan las relaciones durante la adolescencia (Granic, Dishion y Hollenstein, 2003). La llegada de la adolescencia es un momento del ciclo familiar en el que coinciden tres importantes transiciones evolutivas: en el adolescente, en sus padres y en los abuelos; lo que indiscutiblemente repercutirá en el clima familiar.

Desde la infancia, las interacciones sostenidas entre padres e hijos alrededor de las tareas de socialización y de crianza han servido para construir un estilo interactivo, relacional, de funcionamiento familiar, manteniendo una estabilidad y continuidad a lo largo de los años (Oliva, 2006). Entre otros factores, estos estilos-relacionales, educativos, prac-

* Dirección para correspondencia [Correspondence address]:

José M Antón-San Martín. Unidad de Salud Mental Comunitaria.

UGCSM Área Sanitaria Norte de Málaga. Hospital de Antequera. Av. del

Poeta Muñoz Rojas, 29200 Antequera (Málaga, España). E-mail: jmasanmartin@telefonica.net ticas de crianza- tienen el potencial de influir en las trayectorias de salud psicosocial de los hijos. La calidad de las conductas de los padres así como sus creencias se asocian con diferentes resultados de desarrollo.

Dos marcos teóricos diferentes han guiado la investigación en la exploración de las relaciones entre prácticas educativas y resultados en los hijos adolescentes (Darling y Steinberg, 1993). Uno, el categorial, donde se buscan estilos generales de crianza de los hijos para realizar una asignación tipológica. Fue iniciado por las primeras investigaciones de Baumrind (1971) diferenciando tres estilos, el democrático, el autoritario y el permisivo. El primero se define por buscar comunicaciones abiertas con sus hijos, respetan la individualidad y comparten decisiones, a la par que explican las normas y límites a establecer, realizando un control coherente de sus hijos. Los padres autoritarios no fomentan la individualidad e independencia. Buscan el control restrictivo con castigos, amenazas y prohibiciones, a la vez que educan desde criterios rígidos y absolutistas. Los padres con un estilo permisivo evitan hacer uso del control, utilizan pocos castigos, realizan pocas demandas al hijo, y se le permite regular sus propias actividades. Se muestran tolerantes y tienden a aceptar positivamente los impulsos del niño. Maccoby y Martin (1983) ampliaron esta propuesta inicial al establecer una tipología definitiva de 4 estilos parentales: democrático, autoritario, permisivo e indiferente, a partir del cruce de dos dimensiones fundamentales: afecto y control. Este marco teórico ha sido cuestionado debido a que esta tipología y su vinculación al ajuste del adolescente presenta grandes variaciones de acuerdo a características culturales. La relación entre las prácticas de crianza de los hijos y el ajuste varía en función de la etnicidad. (Chao, 2001; Clark, Novak y Dupree, 2002; Whaley, 2000).

El segundo enfoque, el dimensional, focaliza su visión en lo particular más que en lo general, en características conduc- 
tuales de los padres. Se propusieron, inicialmente, tres constelaciones de prácticas paternas que se muestran a lo largo de un continuo (Darling y Steinberg, 1993; Steinberg, Mounts, Lamborn y Dornbusch, 1991; Steinberg, Lamborn y Dornbusch, 1992): la aceptación/implicación, que hace referencia a la calidez e involucración de los padres; el grado de supervisión, que se refiere al control y a la información de la que disponen los padres sobre sus hijos y el nivel de autonomía psicológica permitida, definido como el nivel de fomento de la expresividad así como el uso democrático de las normas disciplinarias. Estas dimensiones han ido aumentando en número hasta llegar a describirse hasta seis: afecto/ comunicación, autonomía, control conductual, control psicológico, revelación de información y humor (Oliva, Parra, Sánchez y López, 2007). Ambos enfoques vinculan el desarrollo infantil a los estilos relacionales mostrados por los padres.

Existe toda una línea de investigación que ha centrado su objetivo en la asociación entre dimensiones comportamentales paternas y la tipología clásica desarrollada por Achenbach (1991). Según ésta, los síntomas en la infancia y adolescencia pueden clasificarse en dos dimensiones: los síntomas internalizantes (ansioso/depresivo, introvertido/depresivo y quejas somáticas) y los síntomas externalizantes (infringir las normas y conducta agresiva) (Ivanova et al., 2007). El control psicológico es más predictivo de trastornos internalizantes (Barber, Olsen y Shagle, 1994; Conger, Conger y Scaramella, 1997; Silk, Morris, Kanaya y Steinberg, 2003) mientras que el control conductual se asocia con los síntomas externalizantes (Galambos, Barker y Almeida, 2003; García, Cerezo, de la Torre, Carpio y Casanova, 2011).

Otros trabajos se han centrado en la relación entre los estilos parentales y las dimensiones particulares con índices específicos de psicopatología como la ansiedad, la depresión, el consumo de drogas, la conducta antisocial, los trastornos de conducta y la conducta suicida. Con respecto a los trastornos de ansiedad Wolfrand, Hempel y Miles (2003) encontraron en una muestra no clínica de estudiantes que el control psicológico parental así como estilo parental autoritario correlacionaron de forma positiva con la despersonalización y la ansiedad, mientras que la dimensión de afecto y la comunicación lo hizo de forma negativa. Los trastornos depresivos se han vinculado con entornos familiares conflictivos (Sheeber, Hops, Alper, Davis y Andrews, 1997), con la dimensión control psicológico (Garber, Robinson y Valentiner, 1997; Martin, Bergen, Roeger y Allison, 2004), con el afecto y comunicación disminuidos (Kim et al., 2003) y con los estilos parentales autoritarios (Sharma, Sharma y Yadava, 2011).

Los estudios cuyo objetivo fue vincular la vulnerabilidad a las conductas suicidas al estilo parental han obtenido asociaciones entre éstas y las dimensiones del control autoritario alto, afecto y comunicación disminuido (Martin y Waite, 1994; Wai y McBrige-Chang, 2001), así como un elevado control psicológico (Palacios, 2010). El estilo parental democrático es el que favorece la menor presencia de conductas suicidas.
La relación entre los trastornos de conducta en la adolescencia y los estilos parentales son los que más interés y estudio han suscitado. Los estilos parentales indiferentes y autoritarios son los que más asociación reciben con los desordenes conductuales (Weiss y Schwarz, 1996). En cuanto a dimensiones conductuales parentales Ary, Ducan, Ducan y Hops (1999), partiendo de la premisa que la conducta antisocial, los comportamientos de riesgo sexual, el fracaso escolar y el consumo de drogas forman una construcción problemática única, obtuvieron como conclusión en su estudio que un escaso y pobre control conductual junto con las asociaciones con compañeros problemáticos era fuertes predictores de este tipo de comportamientos. Kapi, Veltsista, Kavadias, Lekea y Bakoula (2007) hallaron un resultado similar aunque la asociación, junto con esta dimensión conductual parental, era con la ausencia de amistades íntimas. La presencia de niveles altos de control psicológico es la otra variable que más se relaciona con los trastornos de conducta (Bentamcourt y Andrade, 2011; Pettit, Laird, Dodge, Bates y Criss, 2001).

En general, se puede señalar que las investigaciones que analiza la relación entre los estilos parentales y psicopatología en la adolescencia, vinculan el estilo democrático con ajuste y adaptación en los adolescentes. Encuentran una asociación clara entre los trastornos internalizantes, como la ansiedad y la depresión, y el estilo autoritario, así como el estilo parental permisivo o indulgente con síntomas externalizantes (Oliva, Parra y Arranz, 2008). En cuanto a dimensiones conductuales parentales el control psicológico y conductual parecen ser las más relevantes.

Este trabajo pretende analizar, desde una perspectiva transversal, la relación existente entre las dimensiones específicas de los estilos relacionales parentales (afecto/comunicación, autonomía, control conductual, control psicológico, revelación y humor) y la gravedad de la psicopatología presente en una muestra clínica de adolescentes por una parte, y la asociación entre estas dimensiones y la tipología desarrollada por Achenbach (1991) entre síntomas internalizantes versus externalizantes, por otra. El afecto/comunicación, la autonomía, la revelación y el humor serán las dimensiones relacionadas con la estabilidad emocional del adolescente, es decir, a mayor puntuación en ellas menores niveles de gravedad psicopatológica. Por el contrario, la alta presencia de control psicológico y conductual se asociarán con mayor gravedad en los síntomas presentados. En cuanto al segundo objetivo, la la dimensión control conductual se vinculará a la sintomatología externalizante, mientras que la variable control psicológico lo hará con la dimensión internalizante. Se tendrá en cuenta las posibles diferencias que puedan mostrar el género y la edad.

\section{Método}

\section{Participantes}

Se seleccionó una muestra compuesta por 100 adolescentes de ambos sexos (70 chicas y 30 chicos) con edades com- 
prendidas entre los 13 años y 0 meses y 17 años y 11 meses $(M=15.52$ años, $D T=1.62)$, con historia clínica abierta correlativamente en la Unidad de Salud Mental (U.S.M.C.) del Área Sanitaria Norte de Málaga, en el intervalo temporal de dos años consecutivos (Septiembre del 2011 a Septiembre del 2013). La sintomatología que presentaban era heterogénea acudiendo todos con una demanda clínica definida.

Los criterios de exclusión fueron la presencia de un diagnóstico de retraso mental o trastorno mental grave que imposibilitase o dificultase la realización de los cuestionarios y el haber recibido algún tratamiento psicológico en los últimos 6 meses o estar recibiéndolo en el momento de la primera consulta por el posible efecto modificador en la percepción de los estilos parentales.

En la Tabla 1 se recoge una descripción de variables sociodemográficas de la muestra.

Tabla 1. Descripción de variables sociodemográficas.

\begin{tabular}{|c|c|c|c|c|c|}
\hline \multirow{2}{*}{$\overline{\text { Sexo }}$} & \multirow[b]{2}{*}{ Hombre } & \multicolumn{2}{|c|}{ Frecuencia \% } & \multirow{2}{*}{$\frac{M \text { edad }}{14.33}$} & \multirow{2}{*}{$\frac{\text { DT edad }}{1.44}$} \\
\hline & & 30 & 30 & & \\
\hline & Mujer & 70 & 70 & 16.03 & 1.42 \\
\hline & Total & 100 & 100 & 15.52 & 1.62 \\
\hline \multirow{3}{*}{$\begin{array}{l}\text { Rango de } \\
\text { edad }\end{array}$} & 13-14 años & 27 & 27 & & \\
\hline & 15-16 años & 42 & 42 & & \\
\hline & 17-18 años & 31 & 31 & & \\
\hline \multirow{6}{*}{$\begin{array}{l}\text { Curso } \\
\text { Académico }\end{array}$} & $2^{\mathrm{a}} \mathrm{ESO}$ & 15 & 15 & & \\
\hline & $3^{\circ} \mathrm{ESO}$ & 24 & 24 & & \\
\hline & $4^{\circ} \mathrm{ESO}$ & 21 & 21 & & \\
\hline & Bachiller & 18 & 18 & & \\
\hline & Módulos Formativos & 15 & 15 & & \\
\hline & Sin actividad & 7 & 7 & & \\
\hline \multirow[t]{3}{*}{ Convivencia } & Ambos progenitores & 73 & 73 & & \\
\hline & Un progenitor & 23 & 23 & & \\
\hline & Otros familiares & 4 & 4 & & \\
\hline
\end{tabular}

\section{Procedimiento}

Al final de la primera consulta, atendida por alguno de los dos psicólogos clínicos de la U.S.M.C. implicados en el estudio y siguiendo el criterio de edad establecido, se les informaba tanto a los adolescentes como a los familiares acompañantes del objetivo del estudio. Una vez aceptada voluntariamente su participación se firmaba el consentimiento informado y se entregaban los instrumentos de evaluación, siendo recogidos en la segunda consulta.

\section{Instrumentos de evaluación.}

Los participantes cumplimentaron:

- Escala para la evaluación del estilo parental (Oliva, Parra, Sánchez-Queija y López, 2007). Con esta escala se evalúa la percepción que los adolescentes tienen del estilo educativo de sus padres. Es compuesta por 41 ítems, que deben ser puntuados en una escala que abarca desde el 1(totalmente en descuerdo) al 6 (totalmente de acuerdo), agrupados en seis dimensiones: afecto/comunicación (expresión de apoyo y afecto por parte de los padres, disponibilidad y fluidez de la comunicación con ellos), promoción de la autonomía (como alientan a su hijo para que tenga sus propias ideas y tome sus propias decisiones), control conductual (establecimiento de límites y a los intentos de los padres por mantenerse informados sobre el comportamiento de sus hijos e hijas fuera de casa), control psicológico (utilización por parte de los padres de estrategias manipuladoras como el chantaje emocional y la inducción de culpa), revelación (frecuencia con que los adolescentes cuentan a sus padres asuntos personales por propia iniciativa) y humor (en qué medida el adolescente considera que sus padres muestran optimismo y buen sentido de humor). La validación de la misma puede encontrarse en Oliva et a. (2007).

- SCL-90-R. Sympton Checklist 90 Revised. Cuestionario de 90 síntomas. (SCL-90-R, Derogatis, 2002). Es un cuestionario para explorar problemas psicológicos y síntomas psicopatológicos. Dispone de tres niveles de información: tres índices globales (el Índice global de severidad IGS, el Índice positivo de Malestar PSDI, y el Total de síntomas positivos TSP); nueve dimensiones sintomáticas primarias de salud mental (Somatización, Obsesióncompulsión, Sensibilidad interpersonal, Depresión, Ansiedad, Hostilidad, Ansiedad fóbica, Ideación paranoide y Psicoticismo) y un nivel de síntomas discretos. Permite evaluar los síntomas y la intensidad en un paciente en un determinado momento. Consta de 90 ítems a los que se responde sobre la base de una escala de cinco puntos (04). De todos los índices y dimensiones, en este estudio se usará el Índice Global de Severidad (IGS) como medida de gravedad psicopatológica. Hace referencia, como su nombre indica, a una medida generalizada e indiscriminada de la intensidad del sufrimiento psíquico y psicosomático global.

- Inventario de Conducta de los Niños (Child Behavior Checklist, CBCL 4-18) (Achenbach, 1991; Sardinero, Pedreira y Muñíz, 1997). Es una medida de autoinforme que evalúa las competencias psicosociales y distintos problemas de naturaleza conductual y emocional en niños y adolescentes. Se evalúa las conductas problemáticas presentes en los seis últimos meses. Cada opción presenta tres opciones de respuesta: $0=$ la afirmación no es cierta o no es aplicable a mi persona; $1=$ la afirmación es aplicable a mi persona en algunas ocasiones; $2=$ la afirmación se ajusta muy a menudo o frecuentemente a mi persona). A mayor puntuación más presencia de problemas emocionales y comportamentales. Se obtienen varias categorías que se agrupan en dos dimensiones más amplias: síntomas internalizantes (ansioso/depresivo, introvertido/depresivo y quejas somáticas) y síntomas externalizantes (infringir las normas y conducta agresiva). Se agrupará a los sujetos según la pertenencia a una u otra dimensión. 


\section{Análisis de los datos}

Se usaron medidas de tendencia central y dispersión para las variables cuantitativas y distribución de frecuencias para las variables cualitativas. Para analizar las diferencias de las variables cuantitativas en dos grupos independientes se ha aplicado, en el caso paramétrico, la prueba T de Student y en el caso no paramétrico la prueba Mann-Whitney (utilizando como medida de tamaño del efecto la d-Cohen). Para analizar las diferencias en función del rango de edad se ha aplicado, en el caso paramétrico, la prueba ANOVA y, en el caso no paramétrico, la prueba Kruskal Wallis (utilizando como media del tamaño del efecto eta cuadrado parcial). La condición de normalidad se comprobó mediante el test de Shapiro-Wilk. Para establecer las posibles asociaciones y predicciones se realizaron análisis correlacionales (Pearson, Spearman) entre cada una de las variables del estilo parental y el Índice Global de Severidad (IGS) en un primer momento. Con el objetivo de analizar la asociación con las variables del estilo parental se construyeron dos modelos de regresión utilizando, en uno, como variable dependiente el IGS (modelo de regresión lineal) y posteriormente, en otro, como variable dependiente la dimensión internalizante-externalizante (re- gresión lineal). Estos modelos se presentaron crudos y ajustados por sexo y edad (controlando de este modo el posible efecto confusor introducido por estas variables). El nivel de significación establecido fue $p<.05$. El análisis estadístico fue realizado por la Unidad AMEC de FIMABIS usando los paquetes "base", "lsr", "stats" de R software versión 3.1.1 (R Foundation for Statistical Computing, Vienna, Austria; available at http://www.R-project.org).

\section{Resultados}

\section{Estilos parentales e Índice Global de Severidad. Di- ferencias según género y edad.}

Como se aprecia en la Tabla 2 solamente el Índice General de Severidad ha mostrado diferencias significativas en función del sexo $(p=.001)$. Son las adolescentes las que presentan un mayor nivel de intensidad sintomática en sus presentaciones psicopatológicas. Las diferentes variables que componen el estilo parental percibido no han mostrado diferencias significativas.

Tabla 2. Comparación de medias en las diferentes variables del estilo parental e IGS según el género.

\begin{tabular}{|c|c|c|c|c|c|c|}
\hline & $M$ mujer & DT mujer & $M$ hombre & DT hombre & p valor & $d$ Cohen \\
\hline Afecto/Comunicación & 34.10 & 11.95 & 36.66 & 8.26 & $.65 \mathrm{~b}$ & .23 \\
\hline Autonomía & 32.47 & 10.97 & 32.23 & 10.03 & .74 b & .02 \\
\hline Control Conductual & 26.60 & 7.60 & 26.76 & 6.44 & .87 b & .02 \\
\hline Control Psicológico & 27.17 & 10.76 & 25.86 & 10.24 & .52 b & .12 \\
\hline Revelación & 19.14 & 7.18 & 17.33 & 6.56 & $.13 \mathrm{~b}$ & .29 \\
\hline Humor & 23.30 & 8.43 & 24.56 & 6.15 & $.77 \mathrm{~b}$ & .16 \\
\hline IGS & 1.56 & .78 & 1.02 & .67 & $.001^{\mathrm{a}}$ & .71 \\
\hline
\end{tabular}

${ }^{\mathrm{a}}$ Test t-student. ${ }^{\mathrm{b}}$ Test U Mann-Whitney

El IGS presenta diferencias de acuerdo al rango de edad. A medida que aumenta la edad, aumenta el nivel de intensidad sintomática $(\phi=.001)$. Solamente en 1 de las 6 variables que componen el estilo parental percibido aparecen diferen- cias significativas: humor $(p=.03)$, mostrando la variable afecto/comunicación $(p=.05)$ tendencia hacia la significación (Tabla 3).

Tabla 3. Comparación de medias en las diferentes variables del estilo parental e IGS según el rango de edad.

\begin{tabular}{|c|c|c|c|c|c|c|c|c|}
\hline & $M 13-14$ & DT 13-14 & $M 15-16$ & DT 15-16 & $M 17-18$ & DT17-18 & p valor & $\eta 2$ parcial \\
\hline Afecto/Comunicación & 38.44 & 9.46 & 34.40 & 11.24 & 35.09 & 11.34 & $.05 \mathrm{~b}$ & .011 \\
\hline Autonomía & 34.25 & 10.21 & 31.02 & 10.59 & 32.64 & 11.17 & $.40 \mathrm{~b}$ & .003 \\
\hline Control Conductual & 26.70 & 7.60 & 27.23 & 5.89 & 25.80 & 8.62 & $.53 \mathrm{~b}$ & .002 \\
\hline Control Psicológico & 24.44 & 10.19 & 28.92 & 10.27 & 25.90 & 11.08 & $.19 \mathrm{~b}$ & .002 \\
\hline Revelación & 19.44 & 7.21 & 17.54 & 7.31 & 19.90 & 6.43 & $.34 \mathrm{~b}$ & .001 \\
\hline Humor & 29.69 & 7.54 & 21.76 & 7.62 & 23.45 & 7.61 & $.03 \mathrm{~b}$ & .026 \\
\hline IGS & .93 & .73 & 1.49 & .70 & 1.67 & .80 & $.00^{\mathrm{a}}$ & .124 \\
\hline
\end{tabular}

${ }^{a}$ Test t-student. ${ }^{\mathrm{b}}$ Test U Mann-Whitney

Para la exploración de las relaciones entre las diferentes variables del estilo parental y el índice general de severidad se realizaron correlaciones bivariadas. La Tabla 4 presenta los resultados. Se obtienen unas correlaciones muy bajas entre las diferentes variables y la severidad psicopatológica. Destaca la presencia de una correlación negativa en la variable humor $(p=.01)$, lo que indicaría que el optimismo y la pre- sencia del humor en la pauta comunicacional familiar previene la intensidad psicopatológica. Por otra parte, el control psicológico $(p=.02)$ correlaciona de forma positiva con el IGS. 
Tabla 4. Correlaciones entre las variables del estilo parental e IGS.

\begin{tabular}{lcc}
\hline & Coef. & $p$ valor \\
\hline Afecto/Comunicación & -.09 & $.35^{\mathrm{d}}$ \\
Autonomía & -.03 & $.70^{\mathrm{d}}$ \\
Control Conductual & -.03 & $.76^{\mathrm{d}}$ \\
Control Psicológico & .22 & $.02^{\mathrm{c}}$ \\
Revelación & -.03 & $.75^{\mathrm{d}}$ \\
Humor & -.24 & $.01^{\mathrm{d}}$ \\
\hline
\end{tabular}

c Pearson. ${ }^{\mathrm{d}}$ Spearman

En la Tabla 5 se muestran los resultados del análisis de regresión realizado tomando como variable dependiente el IGS. A igualdad de sexo y rango de edad podemos concluir que por cada unidad de incremento de la variable control psicológico, el IGS se incrementa en .01 unidades $(p=.04)$, con una proporción de $16 \%\left(\mathrm{R}^{2}=.16\right)$ de la varianza de la puntuación IGS $(\mathrm{F}=5.83 \mathrm{p}<0.001)$. Con la variable humor ocurre lo contrario: con el incremento disminuye el IGS $(p=.01)$, con una proporción de $18 \%\left(\mathrm{R}^{2}=.18\right)$ de la varianza de la puntuación IGS $(\mathrm{F}=6.395 \mathrm{p}<0.001)$.
Tabla 5. Análisis de regresión lineal bivariante estilo parental e IGS.

\begin{tabular}{lcccc}
\hline & Coef. & $p$ valor & Coef $\bullet$ & $p$ valor \\
\hline Afecto/Comunicación & -.008 & .29 & -.003 & .68 \\
Autonomía & -.005 & .47 & -.004 & .60 \\
Control Conductual & -.008 & .47 & -.007 & .49 \\
Control Psicológico & .01 & .02 & .01 & .04 \\
Revelación & -.005 & .63 & -.008 & .48 \\
Humor & -.03 & .00 & -.02 & .01 \\
\hline
\end{tabular}

- Ajustado por rango de edad y sexo.

\section{Estilos parentales y dimensiones Internalizante y Externalizante}

Del total de la muestra, un $71 \%$ se ubica en la dimensión internalizante (51 mujeres y 20 hombres) y 29 en la externalizante (19 mujeres y 10 hombres) $\left(x^{2}=.53 p<.05\right)$. Los adolescentes que se encuadran en la dimensión externalizante puntúan más alto en la variable control psicológico que los que se encuadran en la dimensión internalizante $(p=.001)$ (Tabla 6). Aquellos adolescentes que presentan conductas agresivas y tendencia a la no aceptación de límites y normas perciben en sus padres como más manipuladores, usando la inducción a la culpa y el chantaje emocional que los adolescentes encuadrados en la dimensión internalizante.

Tabla 6. Comparación de medias en las diferentes variables del estilo parental e IGS según la dimensión Internalizante-Externalizante.

\begin{tabular}{|c|c|c|c|c|c|c|}
\hline & $M$ Inter. & DT Inter. & $M$ Exter. & DT Exter. & $p$ valor & $d$ Cohen \\
\hline Afecto/Comunicación & 35.38 & 10.87 & 33.62 & 11.39 & $.45 \mathrm{~b}$ & .15 \\
\hline Autonomía & 33.53 & 10.13 & 29.62 & 11.54 & $.11 \mathrm{~b}$ & .37 \\
\hline Control Conductual & 26.14 & 7.87 & 27.87 & 5.30 & $.26 \mathrm{~b}$ & .24 \\
\hline Control Psicológico & 25.15 & 10.34 & 30.75 & 10.24 & $.001 \mathrm{a}$ & .54 \\
\hline Revelación & 19.16 & 6.98 & 17.86 & 7.20 & $.43 \mathrm{~b}$ & .18 \\
\hline Humor & 24.07 & 7.67 & 22.74 & 8.20 & $.49 \mathrm{~b}$ & .17 \\
\hline IGS & 1.44 & .77 & 1.29 & .84 & $.42^{\mathrm{a}}$ & .18 \\
\hline
\end{tabular}

a Test t-student. ${ }^{\mathrm{b}}$ Test U Mann-Whitney

Tomando como variable dependiente la dimensión internalizante-externalizante, los resultados que se obtienen en la regresión logística bivariada (Tabla 7) nos dice que los adolescentes con mayor nivel de control psicológico tienen 1.05 veces más riesgo de presentar síntomas externalizantes que los que presentar puntuaciones menores en esa variable. Esto se mantiene en el ajuste por sexo y rango de edad ( $p=.01)$.

Tabla 7. Análisis de regresión logística bivariante estilo parental e Internalizante-Externalizante.

\begin{tabular}{lcccc}
\hline & OR IC 95\% & $P$ valorOR* IC 95\% & $P$ valor \\
\hline Afecto/Comunicación & $.98(.94-1.02)$ & .46 & $.98(.94-1.02)$ & .45 \\
Autonomía & $.96(.92-1.00)$ & .09 & $.96(.92-1.01)$ & .10 \\
Control Conductual & $1.03(.97-1.10)$ & .27 & $1.03(.97-1.0)$ & .28 \\
Control Psicológico & $1.05(1.01-1.1)$ & .01 & $1.05(1.01-1.0)$ & .018 \\
Revelación & $.97(.91-1.03)$ & .39 & $.97(.91-1.04)$ & .47 \\
Humor & $.97(.92-1.03)$ & .43 & $.97(.92-1.03)$ & .43 \\
\hline - Ajustado por rango de edad y sexo.
\end{tabular}

- Ajustado por rango de edad y sexo.

\section{Discusión/ Conclusiones}

El género no parece ser un indicador de diferencias en las dimensiones del estilo parental educativo en nuestra muestra clínica (Smetana, 1995). La intensidad psicopatológica en cambio si discrimina a favor de las adolescentes. Hecho contrario al hallado por Rosa, Parada y Rosa (2014) donde encuentran una menor intensidad sintomática en mujeres utilizando los mismos instrumentos, aunque usando la baremación de muestras no clínicas. Consideramos que las características de las muestras elegidas pueden estar detrás de las diferencias: muestra clínica versus muestra no clínica. Los diversos niveles de desarrollo, las características de personalidad y de familia, así como las variables socioculturales pueden estar en la base. Es aceptada la influencia del género en las manifestaciones psicopatológicas, las cuales se inician en la infancia y se consolidan en la adolescencia (Rojo, 2009), mostrando patrones que se estructuran en la adultez.

En la relación entre estilos parentales percibidos y la gravedad psicopatológica de acuerdo al rango de edad lo hallado: a medida que aumenta el rango de edad más aumenta el índice de severidad, resulta concordante con los estudios de Garaigordobil, Pérez y Mozas (2008) y Rosa et al. (2014). Según estos, la adolescencia tardía presenta unas características diferenciales en cuanto a que problemas se enfrentan así como a que decisiones deben tomar. La intensificación del pro- 
ceso de diferenciación-individuación en el que se encuentran afecta a su equilibrio emocional. De todas las variables del estilo parental percibido solamente el humor aparece depender de la edad. A medida que aumenta la edad disminuye su presencia. Probablemente el mismo proceso de diferenciación-individuación y la distancia emocional y comunicacional que va estableciendose produzca este fenómeno.

El control psicológico al igual que otros estudios realizados, tanto a nivel de psicopatología general (Conger et al., 1997; Oliva et al. 2007; Rosa et al., 2014) como en patologías concretas (Bentamcourt y Andrade, 2011; Garber et al., 1997; Martin et al., 2004; Palacios, 2010; Pettit et al., 2001; Wolfrand et al., 2003) aparece como la variable que más correlaciona no solo con presencia sino también con la intensidad psicopatológica. El uso de retirada del afecto, la inducción de la culpa, el chantaje emocional, como estrategias de control y cambio conductual por parte de los padres, por lo intrusivo y manipulador de las mismas, son las que provocan más alteraciones emocionales en los menores. El humor, entendido como una actitud relacional por parte de los padres donde prima la serenidad, la alegría y el optimismo creando un clima familiar que favorece, no solo la comunicación interna sino los procesos de socialización de los adolescentes (Darling y Steinberg, 1993; Oliva, Parra y Arranz, 2008) aparece como variable protectora en nuestro estudio.

La mayoría de los estudios realizados (Barber et al., 1994; Galambos et al., 2003; García et al., 2011; Rosa et al., 2014; Silk et al., 2003) asocian la dimensión de control psicológico con sintomatología internalizante mientras que la externalizante la correlacionan con el control conductual. En nuestro estudio es el control psicológico la variable donde más alto puntúan nuestros adolescentes con sintomatología externalizante, incluso a mayor control psicológico más riesgo de síntomas de este tipo. Contradice los hallazgos realizados hasta

\section{Referencias}

Achenbach, T. (1991). Manual for the Youth Self-Report and 1991 YSR profile. Burlington, V.T: University of Vermont.

Ary, D., Duncan, T., Duncan, S. \& Hops, H. (1999). Adolescent problem behavior: the influence of parents and peers. Behaviour Research and Therapy, 37, 217-230.

Barber, B., Olsen, J. \& Shagle, S. (1994). Association between parental psychological and behavioral control and youth internalized and externalized. Behavior Child Development, 65, 1120-1136.

Baumrind, D. (1971). Current Patterns of Parental Authority. Developmental Psychology Monograph, 4(1), 1-103.

Betancourt, D. y Andrade, P. (2011). Control parental y problemas emocionales y de conducta en adolescentes. Revista Colombiana de Psicología, 20(1), 27-41.

Carter, B. y McGoldrick, M. (1980). The changing Family life cycle. A framework for family therapy. Gardner Press. New York.

Chao, R. (2001). Extending Research on the Consequences of Parenting Style for Chinese Americans and European Americans. Child Development, 72(6), 1832-1843.

Clark, R., Novak, J. y Dupree, D. (2002). Relationship of perceived parenting practices to anger regulation and coping strategies in AfricanAmerican adolescents. Journal of Adolescence, 25,373-384. doi:10.1006/jado.2002.0482 ahora. Podemos suponer que una vez instaurado un nivel de psicopatología en el adolescente se producen, en la convivencia diaria, rigidificaciones en los estilos relacionales paternos en cuanto al uso de la manipulación emocional como elemento de control, provocando, a su vez, reacciones emocionales y conductuales intensas en los hijos.

Las limitaciones observadas en este estudio son varias. Por una parte, el uso de un solo autoinforme por sujeto y no dos, uno por cada padre, se puede percibir como una limitación del estudio. El haber realizado esta elección, se basa en el hecho que la comparación entre los dos estilos muestran que las concurrencias son más frecuentes que las discrepancias, llegando a una coincidencia del 75\% (Musitu y García, 2004; Oliva et al., 2007). Y por otro, la elección realizada de usar solo como única fuente de la información la obtenida del adolescente. Existen estudios que indican que la percepción del adolescente sobre el funcionamiento de sus padres suele ser más objetiva y correlacionar más con observadores externos que la de los padres y madres, que suele estar más sesgada por la influencia de la deseabilidad social (Gonzales, Cauce y Mason, 1996; Iglesias y Romero, 2009).

Sería necesario continuar las investigaciones usando muestras definidas como clínicas. Es decir, adolescentes que ya se encuentran inmersos en procesos de sufrimiento ya que parece que este criterio puede apuntar a hallazgos distintos a los encontrados usando muestras poblacionales comunitarias. Lo diferente de las muestras usadas así como los diversos instrumentos de evaluación utilizados puede justificar esta desigualdad. Consideramos que el análisis de estas dimensiones en muestras de adolescentes con psicopatología de diferente tipo aportará nueva información sobre el peso de cada una de ellas, siendo de gran utilidad en los procesos de cambio terapéutico a implementar con los adolescentes y sus familias.

Conger, K., Conger, R. y Scaramella, L. (1997). Parents, sibling, psychological control and adolescent adjustment. Journal of Adolescent Research, 12, 113-38.

Darling, N. y Steinberg, L. (1993). Parenting style as context: an integrative model. Psychological Bulletin, 113(3), 487-496.

Derogatis, L. (2002). SCL-90-R. Cuestionario de Sintomas. TEA Ediciones. Madrid.

Falicov, C. (comp.). (1991). Transiciones en la Familia. Continuidad y cambio en el ciclo de vida. Amorrortu editores. Buenos Aires.

Galambos, N., Barker, E. \& Almeida, D. (2003). Parents do matter: Trajectories of change in externalizing and internalizing problems in early adolescence. Child Development, 74(2), 578-594. DOI: 0009 3920/2003/7402-0017.

Garaigordobil, M., Pérez, J. y Mozas, M. (2008). Self-concept, self-esteem and psychopathological symptoms. Psicothema, 20, 114-123.

Garber, J, Robinson, N, y Valentiner, D. (1997). The relation between parenting and adolescent depression: Self as a mediator. Journal of Adolescent Research, 12, 12-33.

García, M., Cerezo, T., de la Torre M., Carpio, M. y Casanova P. (2011) Prácticas educativas paternas y problemas internalizantes y externalizantes en adolescentes españoles. Psicothema, 23(4), 654-659.

Gonzales, N., Cauce, A. y Mason, C. (1996). Interobserver agreement in the assessment of parental behavior and parent-adolescent conflict: Afri- 
can-American mothers, daughters and independent observer. Child Development, 67, 1483-1498.

Granic, I., Dishion, T. y Hollenstein, T. (2003). The family ecology of adolescence: A dynamic systems perspective on normative development. En Adams, G. y Berzonsky, M. (Ed.). The Blackwell Handbook of Adolescence. UK: Blackwell. Oxford.

Iglesias, B. y Romero, E. (2009). Estilos parentales percibidos, psicopatología y personalidad en la adolescencia. Revista de Psicopatología y Psicología Clínica. 14(2), 63-77.

Ivanova, M., Achenbach, T., Rescorla, L., Dumenci, L., Almqvist, F., Bilenberg, N.,...Verhulst, F. (2007). The generalizability of the Youth SelfReport Syndrome Structure in 23 societies. Journal of Consulting and Clinical Psychology, 75, 729-738. doi:10.1037/0022-006X.75.5.729.

Kapi, A., Veltsista, A., Kavadias, G., Lekea, V. y Bakoula, Ch. (2007). Social determinants of self-reported emotional and behavioral problems in Greek adolescents. Social Psychiatry and Psychiatric Epidemiology. 42 (7), 594-598.

Kim, I., Ge, X., Brody, G., Conger, R., Gibbons, F. y Simons, R. (2003). Parenting behaviors and the occurrence and co-occurrence of depressive symptoms and conduct problems among african american children. Journal of Family Psychology, 17(4), 571-583.

Maccoby, E. y Martin, J. (1983). Socialization in the context of the family: parent-child interaction. En Hetherington, E. y Mussen, P. (Eds.). Handbook of Child Psychology, Vol. IV : Socialization, Personality and Social Development. Wiley. New York.

Martin, G., Bergen, H., Roeger, L. y Allison, S. (2004). Depression in young adolescents. Investigations using 2 and 3 factor versions of the Parental Bonding Instrument. The Journal of Nervous and Mental Disease, 192(10), 650-657. doi:10.1097/01.nmd.0000142028.10056.c6

Martin, G. y Waite, S. (1994). Parental bonding and vulnerability to adolescent suicide. Acta Psychiatrica Scandinavica, 89, 246-54.

Musitu, G. y García, F. (2004). ESPA29, Escalas de estilos de socialización en la adolescencia $\left(2^{\circ}\right.$ ed.). Madrid. TEA ediciones.

Oliva, A. (2006). Relaciones familiares y desarrollo adolescente. Anuario de Psicología, 37(3), 209-216.

Oliva, A., Parra, A. y Arranz, E. (2008). Estilos relacionales parentales y ajuste adolescente. Infancia y Aprendizaje, 31,93-106.

Oliva, A., Parra, A., Sánchez, L. y López F. (2007). Estilos educativos materno y paterno: Evaluación y relación con el ajuste adolescente. Anales de Psicología, 23(1), 49-56.

Palacios, J. (2010). Influencia de los estilos parentales en adolescentes que han intentado suicidarse. Revista de Psicoterapia. 84, 85-93.

Pettit, G., Laird, R., Dodge, K., Bates, J. y Criss, M. (2001). Antecedents and behavior-problem outcomes of parental monitoring and psychological control in early adolescence. Child Development, 72(2), 583-598. doi:00093920/2001/7202-0017.

Rojo, N (2007). Diferencias de género en la psicopatología de adolescentes. En Pérez S., Rojo N. e Hidalgo A. (Coords.) (2007). La salud mental de las personas jóvenes en España. Revista de Estudios de Juventud. 84.

Rosa, A., Parada, J. y Rosa, A. (2014). Síntomas psicopatológicos en adolescentes españoles: Relación con los estilos parentales percibidos y autoestima. Anales de Psicología 30 (1), 133-142.

Sardinero, E., Pedreira, J. y Muñiz, J. (1997). El cuestionario CBCL de Achenbach: Adaptación española y aplicaciones clínicoepidemiológicas. Clínica y Salud, 8(3), 447-80.

Sharma, M., Sharma, N. y Yadava, A. (2011). Parental styles and depression among adolescents. Journal of the Indian Academy of Applied Psychology, 37(1), 60-68

Sheeber, L., Hops, H., Alpert, A., Davis, B. y Andrews, J. (1997). Family Support and Conflict: Prospective Relations to Adolescent Depression. Journal of Abnormal Child Psychology, 25, 333-344.

Smetana, J. (1995). Parenting style and conceptions of parental authority during adolescence. Child Development, 66, 299-316. doi:00093920/2002/7302-0015.

Steinberg, L., Mounts, N., Lamborn, S. y Dornbusch, S. (1991). Authoritative parenting and adolescent adjustment across various ecological niches. Journal of Research on Adolescence, 1, 19-36.

Steinberg, L., Lamborn, S. y Dornbusch, S. (1992). Impact of parenting practices adolescent achievement: authoritative parenting, school involvement, and encouragement to succeed. Child Development, 63, 12661281.

Silk, J., Morris, A., Kanaya, T. y Steinberg, L. (2003). Psychological Control and Autonomy Granting: Opposite Ends of a Continuum or Distinct Constructs?. Journal of Research on Adolescence, 13, 113-128.

Wai K. y McBride-Chang C (2001). Suicidal ideation, parenting style, and family climate a among Hong Kong adolescents. International Journal of Psychology, 36(2), 81-87. doi: 10.1080/00207590042000065.

Weiss, L. y Schwarz, J. (1996). The relationship between parenting types and older adolescent personality, academic achievement, adjustment and substance use. Child Development, 67, 2101-2111.

Whaley, A. (2000). Sociocultural differences in the developmental consequences of the use of physical discipline during childhood for African Americans. Cultural Diversity and Ethnic Minority Psychology, 6, 5-12.

Wolfradt, U., Hempel, S. y Miles, J. (2003). Perceived parenting styles, depersonalization, anxiety and coping behavior in adolescents. Personality and Individual Differences, 34, 521-532.

(Articulo recibido: 27-08-2014; revisado: 08-10-2014; aceptado: 09-12-2014) 